\title{
At Physiologic Albumin/Oleate Concentrations Oleate Uptake by Isolated Hepatocytes, Cardiac Myocytes, and Adipocytes Is a Saturable Function of the Unbound Oleate Concentration
}

\author{
Uptake Kinetics Are Consistent with the Conventional Theory
}

\author{
Dario Sorrentino, Richard B. Robinson, Chi-Li Kiang, and Paul D. Berk \\ Polly Annenberg Levee Hematology Center and the Hepatic Research Group (Department of Medicine), Mount Sinai \\ School of Medicine, New York, New York 10029; and Department of Pharmacology, Columbia University \\ College of Physicians and Surgeons, New York, New York 10032
}

\begin{abstract}
To reexamine the role of albumin in cellular uptake of long chain fatty acids, we measured $\left[{ }^{3} \mathbf{H}\right]$ oleate uptake by isolated hepatocytes, adipocytes, and cardiac myocytes from incubations containing oleate/albumin complexes at molar ratios from 0.01:1 to 2:1. For each ratio the uptake was studied over a wide range of albumin concentrations. In all three cell types and at any given oleate/albumin ratio, the uptake appeared saturable with increasing concentrations of oleate:albumin complexes despite the fact that the unbound oleate concentration for each molar ratio is essentially constant. However, the " $K_{m}$ " but not the " $V_{\max }$ " of these pseudosaturation curves was influenced by substrate availability. At low albumin concentrations, uptake velocities did not correlate with unbound oleate concentrations. However, observed and expected uptake velocities coincided at albumin concentrations approaching physiologic levels and were a saturable function of the oleate/albumin ratios and the consequent unbound oleate concentrations employed. Hence, under the experimental conditions employed in this study using a variety of suspended cell types, oleate uptake kinetics were consistent with the conventional theory at physiologic concentrations of albumin.
\end{abstract}

\section{Introduction}

The uptake of albumin bound ligands such as fatty acids, bilirubin, bile acids, hormones, and many xenobiotics is a complex process involving several discrete but interrelated steps: namely, dissociation from albumin, transport across the plasma membrane, binding to cytoplasmic proteins, metabolism, and/or excretion (1). Even though in many tissues, such as the heart and adipose tissue, the capillary endothelium represents a barrier to ligand extraction by the parenchymal cells, uptake rates in vivo are substantial $(2,3)$. In the liver, the fenestrations connecting the sinusoid with the space of Disse allow free contact between albumin/ligand complexes in the circulation and the hepatocellular surface. In either case, how-

Address reprint requests to Dr. Sorrentino, Department of Medicine, Box 1079, Mount Sinai School of Medicine, 1 Gustave Levy Place, New York, NY 10029.

Received for publication 12 December 1988 and in revised form 14 June 1989.

J. Clin. Invest.

(c) The American Society for Clinical Investigation, Inc.

$0021-9738 / 89 / 10 / 1325 / 09 \$ 2.00$

Volume 84, October 1989, 1325-1333 ever, cells do not appear to internalize albumin at a significant rate (4-6). Therefore, dissociation of the albumin ligand complex must precede ligand uptake.

According to the conventional theory of cellular uptake, only unbound ligand participates in the uptake process (7-9). Since the fraction of the total ligand present at any time in unbound form is extremely small, the dissociation rate of ligand/albumin complexes would have to be fast enough to match ligand uptake rates. Thus, it is assumed that virtually instantaneous equilibration occurs between bound and unbound ligand. The conventional theory was first challenged by Baker and Bradley (10), who concluded that hepatic sulfobromophthalein (BSP) ${ }^{1}$ uptake in vivo significantly exceeded what could be accounted for by the very small concentration of nonalbumin-bound BSP. Similar questions were posed by Bloomer et al. (11) and Anwer et al. (12). Although provocative, these issues went almost unnoticed for ten years until more recent observations confirmed and expanded the controversy. In 1981, virtually simultaneously, Weisiger et al. and Forker and Luxon highlighted other apparent pitfalls of the traditional theory $(13,14)$. They reported, inter alia, that hepatic uptake velocity for several ligands $(a)$ exceeded the reported in vitro dissociation rates of the corresponding albumin/ligand complexes; $(b)$ appeared to be a function of total rather than unbound ligand; $(c)$ was apparently competitively inhibited by the addition of uncomplexed albumin; and $(d)$ appeared to saturate with increasing albumin rather than ligand concentrations (13-18). These authors interpreted their kinetic data as indicating some form of facilitated dissociation of the albumin ligand complexes, mediated either by a specific albumin receptor or by a nonspecific interaction between albumin and the cell surface. Even though many studies have confirmed the validity of these observations, employing a variety of albumin bound ligands and many experimental settings (19-25), their interpretation has been the cause of much confusion and uncertainty. While accumulating evidence has made it unlikely that the hepatocyte surface bears a discrete albumin receptor (26-30), aspects of the relevant data still seem inconsistent with the conventional model $(31,32)$. Cellmediated dissociation of albumin ligand complexes (33), the presence of a large unstirred water layer in the space of Disse (34) and dissociation limited availability of substrate (27, 35-37) are the principal alternative theories proposed to explain the observed kinetics.

1. Abbreviations used in this paper: BSP, sulfobromophthalein; $\mathrm{HH}$, Hanks' solution with $10 \mathrm{mM}$ Hepes; KRH, Krebs-Ringer Hepes buffer. 
Although this issue has been the subject of intensive investigation, one fundamental question remains unanswered; that is, what is the physiologic relevance of the observed phenomena? Many of the studies in this area have employed low, nonphysiologic concentrations of albumin and ligand. Accordingly, the aims of the present study were to determine: $(a)$ whether the traditional theory can explain the kinetics of oleate uptake by isolated cells using albumin/ligand concentrations occurring in vivo, and $(b)$ whether, in this respect, patterns of uptake kinetics qualitatively similar to those observed with liver cells can be obtained using cell types which in vivo interact with the interstitial, rather than intravascular, albumin pool. For these purposes, we have used a well validated rapid filtration technique to study the velocity of $\left[{ }^{3} \mathrm{H}\right]-$ oleate uptake by isolated suspensions of hepatocytes, adipocytes and cardiac myocytes. The data demonstrate that at physiologic albumin/oleate concentrations, oleate uptake into isolated suspensions of all three cell types is a saturable function of the unbound oleate concentration, and is therefore consistent with the conventional theory. At low, nonphysiologic albumin/ligand concentrations uptake kinetics deviate from the expected behavior and require an alternative explanation. Preliminary reports of these findings have been published in abstract form $(38,39)$.

\section{Methods}

Materials. 9,10-[ $\left[{ }^{3} \mathrm{H}\right]$ Oleic acid $(2-6 \mathrm{Ci} / \mathrm{mmol})$ was obtained from New England Nuclear (Boston, MA); nonradioactive oleate, trypsin inhibitor and bovine serum albumin (fraction V, essentially fatty acid free) from Sigma Chemical Co. (St. Louis, MO); and collagenase from Boehringer Mannheim (Indianapolis, IN).

Cell isolation. Hepatocytes and adipocytes were obtained from male Sprague-Dawley rats (55-60 d old) by collagenase perfusion and digestion, respectively, as previously reported $(40,41)$. Briefly, after an initial perfusion with Hanks' balanced salt solution, $\mathrm{Ca}^{2+}$ and $\mathbf{M g}^{2+}$ free, livers were perfused for $15 \mathrm{~min}$ at $37^{\circ} \mathrm{C}$ with the same solution containing $\mathrm{Ca}^{2+}, 0.05 \%$ collagenase, and $0.001 \%$ trypsin inhibitor. Hepatocytes were then separated by gentle scraping, differential centrifugation and filtration through nylon mesh. Cells were resuspended $(\sim 2$ $\left.\times 10^{6} / \mathrm{ml}\right)$ in Hanks' solution, containing $10 \mathrm{mM}$ Hepes pH $7.4(\mathrm{HH}$ buffer). Hepatocyte preparations met previously established viability criteria (40). In particular, viability was $\geq 90 \%$ when assessed by the trypan blue exclusion test. Adipocytes were isolated by digestion of epididymal fat pads with collagenase for $45 \mathrm{~min}$ at $37^{\circ} \mathrm{C}$. After filtration through nylon mesh the cells were washed and resuspended in Krebs-Ringer Hepes pH 7.4 (KRH buffer) at a concentration of $\sim 1$ $\times 10^{6}$ cells $/ \mathrm{ml}$ and viability assessed by the insulin-stimulated lipogenesis assay as previously reported (42). By this technique there was no loss of viability between freshly isolated adipocytes and those incubated for up to $2 \mathrm{~h}$. Cardiac myocytes were isolated from dogs (1-2-yrold beagles) by collagenase perfusion in the presence of $\mathrm{CaCl}_{2}$ and resuspended in $\mathrm{HH}$ buffer $\left(\sim 0.8 \times 10^{6} \mathrm{cells} / \mathrm{ml}\right)$ until use $(43,44)$. When tested, $\sim 85-90 \%$ of cardiac myocytes excluded trypan blue; of these up to $80 \%$ retained the rod shape. Ultrastructural, pharmacologic, and electrophysiologic features of this preparation have been reported elsewhere (43).

Oleate uptake studies. The uptake of oleate was studied in the three different cell types by a rapid filtration technique as described in detail elsewhere $(40,41,44,45) .9,10\left[{ }^{3} \mathrm{H}\right]$ Oleic acid, measured amounts of nonradioactive oleate, and albumin were dissolved in the same buffer in which cells were resuspended ( $\mathrm{HH}$ for hepatocytes and cardiac myocytes and KRH for adipocytes) at various oleate/albumin molar ratios and concentrations (see Results). At zero time $500 \mu \mathrm{l}$ (except where specified) of the appropriate oleate/albumin solution was added under stirring to $100 \mu \mathrm{l}$ (hepatocytes and cardiac myocytes) or $50 \mu \mathrm{l}$ (adipocytes) of cell suspension in a 15-ml polystyrene tube (Sarstedt, Inc., Princeton, NJ). At given intervals thereafter uptake was blocked with $5 \mathrm{ml}$ of an ice-cold $0.1 \%$ albumin/phloretin $(200 \mu \mathrm{M}$ for hepatocytes and myocytes and $400 \mu \mathrm{M}$ for adipocytes) stop solution prepared in the same buffer in which oleate/albumin had been dissolved; the mixture was immediately filtered and the filters (GF/C from Whatman, Maidstone, England) counted by liquid scintillation spectrometry. Zero time uptake values were determined by adding stop solution to cells before adding the oleate/albumin solution. Appropriate filter blanks were routinely determined and substracted from uptake values. Initial oleate uptake velocity was estimated as the slope of the initial linear portion of the cumulative uptake curve. We have previously shown that the technique employed in this study affords an accurate measure of initial oleate influx rates in these different cell types (40, 41, 44).

Unbound oleate concentrations. For each oleate/albumin molar ratio $(\nu)$ studied, the equilibrium concentration of unbound oleate was calculated by the stepwise equilibrium method as described by Spector et al. $(46,47)$, and programmed for digital computer by Wosilait and Nagy (48). Since the pertinent equations constitute a simplifying model that does not take into account cooperativity effects and assumes that the reactants have ideal activities we sought to confirm these calculated values (see Table I), by determining actual unbound oleate concentrations at each $\nu$ up to $2: 1$ employing the $n$-heptanewater partition method originally described by Goodman (49). Experimentally determined results $(n=8)$ were in good agreement with calculated values; when measured values over a wide range of $\nu$ were plotted against calculated values, the slope did not differ from unity $(P$ $>0.1)$, and the data were highly correlated $(r=0.996)$. Furthermore, additional studies indicated that unbound oleate concentrations were essentially constant (within the limits of experimental error) for any tested $\nu$ as predicted by the stepwise equilibrium method and the ratio of the unbound oleate concentration measured at the lowest albumin concentration $(16 \mu \mathrm{M})$ to that measured at the highest albumin concentration $(600 \mu \mathrm{M})$ was $1.0 \pm 0.1$.

Data analysis. Where indicated data were fitted directly to the Michaelis Menten equation using the Simulation, Analysis and Modeling (SAAM) program of Berman and Weiss (50) on the IBM mainframe computer system of the City University of New York. Statistical differences between groups were assessed by the unpaired $t$ test.

Table I. "V $V_{\max }$ "Values for $\left[{ }^{3} \mathrm{H}\right]$ Oleate Uptake at Fixed Oleate/Albumin Molar Ratios

\begin{tabular}{llccc}
\hline & & \multicolumn{3}{c}{ “ $V_{\max }$ " } \\
\cline { 4 - 5 } Oleate/albumin & $\begin{array}{c}\text { Unbound } \\
\text { Oleate } \\
\text { conc.* }\end{array}$ & Hepatocytes & Adipocytes & $\begin{array}{c}\text { Cardiac } \\
\text { myocytes }\end{array}$ \\
\cline { 4 - 5 } \multicolumn{1}{c}{$\nu$} & $\mu \mathrm{M}$ & & & \\
$0.01: 1$ & 0.00061 & - & $40 \pm 17$ & $15 \pm 2$ \\
$0.025: 1$ & 0.0015 & - & - & $32 \pm 5$ \\
$0.1: 1$ & 0.0065 & $9 \pm 1$ & $385 \pm 26$ & $139 \pm 8$ \\
$0.25: 1$ & 0.018 & $21 \pm 2$ & $1,129 \pm 58$ & $162 \pm 8$ \\
$0.4: 1$ & 0.031 & $42 \pm 5$ & - & $192 \pm 4$ \\
$0.5: 1$ & 0.043 & $46 \pm 4$ & - & - \\
$1: 1$ & 0.12 & $50 \pm 2$ & $1,471 \pm 58$ & $261 \pm 16$ \\
$2: 1$ & 0.43 & $95 \pm 14$ & $1,331 \pm 132$ & $399 \pm 27$ \\
& & & & \\
\hline
\end{tabular}

Uptake values shown in Fig. 1 were fitted to the Michaelis Menten equation as described in Methods. Data are expressed as mean $\pm \mathrm{SD}$ $(n=3)$ in picomoles/minute $/ 5 \times 10^{4}$ cells.

* Calculated as described in Methods. 


\section{Results}

Effects of concentration of oleate/albumin complex. Despite the fact that the equilibrium concentration of unbound oleate in a solution containing oleate and albumin is essentially constant for a given value of $\nu$, and virtually independent of the absolute concentrations of oleate and albumin in the solution (46), at each $\nu$ studied and for each cell type, increasing the concentration of oleate/albumin produced apparent saturation of oleate uptake (Fig. 1). This phenomenon, similar to that previously reported in the isolated perfused rat liver (13), was equally observed with isolated suspensions of hepatocytes (Fig. $1 A$ ), adipocytes (Fig. $1 B$ ), and cardiomyocytes (Fig. $1 C$ ). For each cell type, the uptake vs. concentration curve for each $\nu$ could be fitted to the Michaelis Menten equation; although the " $V_{\max }$ ' $\mathrm{s}$ ", ${ }^{2}$ not surprisingly, differ between the cell types, the results are qualitatively similar. For each cell type, the " $V_{\max }$ ' $s$ " increased progressively as $\nu$ was increased from $0.01: 1$ to 2:1 (Table I). Since the maximal uptake velocity observed at a given value of $\nu$ could be exceeded merely by increasing $\nu$, the hyperbolic uptake vs. concentration curves and relative " $V_{\max }$ ' $s$ " observed in this setting clearly do not reflect actual saturation of a transport step in cellular oleate uptake. These data confirm prior observations by others $(13,25)$ but provide the essential background for the observations that follow.

Correlation between uptake and unbound oleate concentration. Since each of the curves described above was determined in a setting in which the equilibrium unbound oleate concentration was essentially constant (i.e., using a fixed $\nu$ ) (46), there was apparently a lack of correlation between uptake and the equilibrium concentration of unbound ligand over the broad range of absolute oleate/albumin concentrations employed in each study. A further analysis of the data was conducted to examine specifically the relationship between equilibrium unbound oleate concentration and oleate uptake when the concentrations of oleate/albumin were within the physiologic range (500-700 $\mu \mathrm{M}$ ). Note (Fig. 1) that, for all three cell types and all values of $\nu$ studied, " $V_{\max }$ " uptake rates occurred at or near physiologic concentrations of oleate/albumin.

The calculated equilibrium unbound oleate concentrations at $\nu$ of 0.4:1, 0.25:1 and 0.1:1 are 0.031 $\mu \mathrm{M}, 0.018 \mu \mathrm{M}$, and $0.0065 \mu \mathrm{M}$, respectively (46-48). If uptake were driven principally by the unbound oleate concentration, decreasing $\nu$ from $0.4: 1$ to $0.25: 1$, from $0.25: 1$ to $0.1: 1$, or from $0.4: 1$ to $0.1: 1$ should produce a decrease in uptake velocity of 42,64 , or $79 \%$, respectively, corresponding to the decrease in estimated equilibrium unbound oleate concentration. In Fig. 2, the initial portions of the pseudosaturation curves for oleate uptake by hepatocytes at $\nu$ of $0.4: 1,0.25: 1$, and $0.1: 1$ are reproduced, with lines showing the effects on uptake of decreasing $\nu$ at particular absolute concentrations of oleate. The expected lin-

2 . In this context when " $V_{\max }$ " and " $K_{\mathrm{m}}$ " are employed in quotation marks, they do not have the usual meaning of these kinetic parameters but rather denote the total concentration of albumin (" $K_{m}$ ") at which ligand uptake is half of its maximal value (" $V_{\max }$ ") at a particular fixed ligand/albumin molar ratio (see for example Fig. 1). When they are not enclosed in quotation marks $V_{\max }$ and $K_{\mathrm{m}}$ have the usual meaning for a ligand transported by a saturable membrane transport system. $V_{\max }$ refers to the maximal ligand uptake velocity and $K_{\mathrm{m}}$ to the unbound concentration of ligand at which uptake is half its maximal value (see for example Fig. 4). ear relationship ${ }^{3}$ between changes in $\nu$ (and consequent unbound oleate concentration) and oleate uptake velocity is not observed at low concentrations of oleate/albumin. For example, as illustrated in Fig. 2, at a final total oleate concentration of $10 \mu \mathrm{M}$, increasing albumin from 25 to $40 \mu \mathrm{M}$, thereby decreasing $\nu$ from $0.4: 1$ to $0.25: 1$, decreases uptake by only $17 \%$; a further increase in albumin to $100 \mu \mathrm{M}$ ( $\nu$ decreasing from $0.25: 1$ to $0.1: 1$ ) results in an additional decrease in oleate uptake velocity of $23 \%$. Overall, as $\nu$ decreases from $0.4: 1$ to $0.1: 1$, uptake velocity decreases by $37.5 \%$. Hence, at $10 \mu \mathrm{M}$ oleate, the observed decreases in uptake velocity with decreasing values of $\nu$ are substantially smaller than the corresponding decreases in the equilibrium unbound oleate concentration. However, the discrepancies between observed uptake velocities and those predicted from the equilibrium unbound oleate concentrations are progressively reduced as the concentration of oleate/albumin complex increases, (see for example in Fig. 2 the relationship between uptake values at different $\nu$ for 50 or $100 \mu \mathrm{M}$ oleate concentration) until at oleate/albumin concentrations yielding maximal uptake velocities (" $V_{\max }$ 's") for the $\nu$ in question, the observed and predicted uptake velocities coincide (Fig. 3).

In the example illustrated in Fig. 2 " $V_{\max }$ " values are $41.8 \pm 4.6,21.4 \pm 1.7$, and $8.7 \pm 0.99 \mathrm{pmol} / \mathrm{min}$ per 50,000 cells at $\nu=0.4: 1,0.25: 1$, and $0.1: 1$, respectively (Table I). Thus, at physiologic concentrations of oleate/albumin, the maximal uptake velocities decrease by factors of $49 \%(\nu=0.4: 1 \rightarrow$ $0.25: 1), 60 \%(\nu=0.25: 1 \rightarrow 0.1: 1)$, and $79 \%(\nu=0.4: 1 \rightarrow$ $0.1: 1)$, agreeing closely with the results predicted on the basis of changes in the unbound oleate concentrations. Virtually identical results were obtained with isolated cardiac myocytes and adipocytes (Fig. 3), thus suggesting that the observed kinetics are the result of a phenomenon not unique to the liver cell. These studies indicate that, at physiologic oleate/albumin concentrations, oleate uptake by suspensions of hepatocytes, adipocytes, and cardiac myocytes is in fact a saturable function of the unbound oleate concentration, as predicted by the conventional theory. The lack of correlation between uptake velocity and unbound oleate concentration is observed only at nonphysiologic conditions with respect to oleate and albumin concentrations, concentrations, in fact, unlikely to occur in vivo.

" $V_{\max }$ " values vs. unbound oleate concentrations. When the " $V_{\max }$ " values obtained for each $\nu$ were plotted versus the corresponding unbound oleate concentrations, a rectangular hyperbola consistent with Michaelis-Menten kinetics was observed for each cell type (Fig. 4). Estimated parameters were, $V_{\max }: 105 \pm 13,1590 \pm 170$, and $422 \pm 30 \mathrm{pmol} / \mathrm{min}$ per $5 \times 10^{4}$ cells; $K_{\mathrm{m}}: 74 \pm 13,21 \pm 4$, and $30 \pm 4 \mathrm{nM}$ for hepatocytes, adipocytes, and cardiac myocytes, respectively $(n=3-4$, mean $\pm S D)$. These data indicate that at physiologic oleate/al-

3. For a saturable intrinsic membrane transport system, the conventional model predicts a linear relationship between uptake velocity and unbound ligand concentration at unbound ligand concentrations well below the $K_{\mathrm{m}}$ for the transported ligand, beyond which the relationship clearly becomes nonlinear. Indeed, once $V_{\max }$ for ligand uptake is reached at high concentrations of unbound ligand, no correlation is expected between uptake velocity and further changes in unbound ligand. For these reasons, the molar ratios in question (Figs. 2 and 3) were selected to provide unbound ligand concentrations below $K_{\mathrm{m}}$ as established in preliminary studies. 

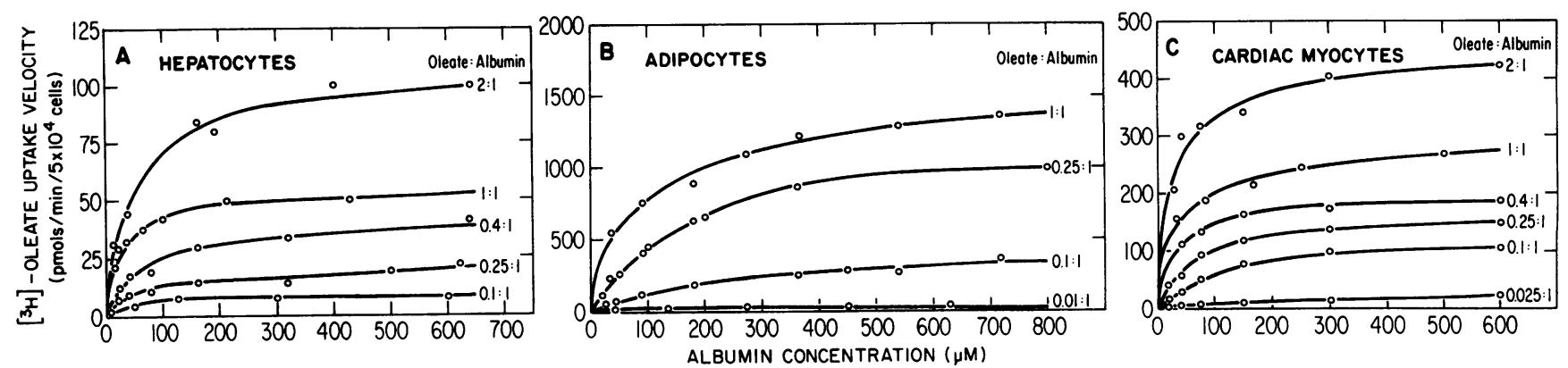

Figure 1. $\left[{ }^{3} \mathrm{H}\right]$ Oleate uptake velocity by isolated hepatocytes $(A)$, adipocytes $(B)$, and cardiac myocytes $(C)$ as a function of the oleate/albumin ratio $(\nu)$ and albumin concentration. Although uptake appears saturable for any given $\nu$, the " $V_{\max }$ " is a function of the $\nu$ employed (Table $\mathrm{I}$ ) and can be exceeded merely by moving to a higher $\nu$, indicating that these curves do not reflect true saturation of a transport step.

bumin concentrations oleate transport across the plasma membrane is a saturable function of the unbound oleate concentration.

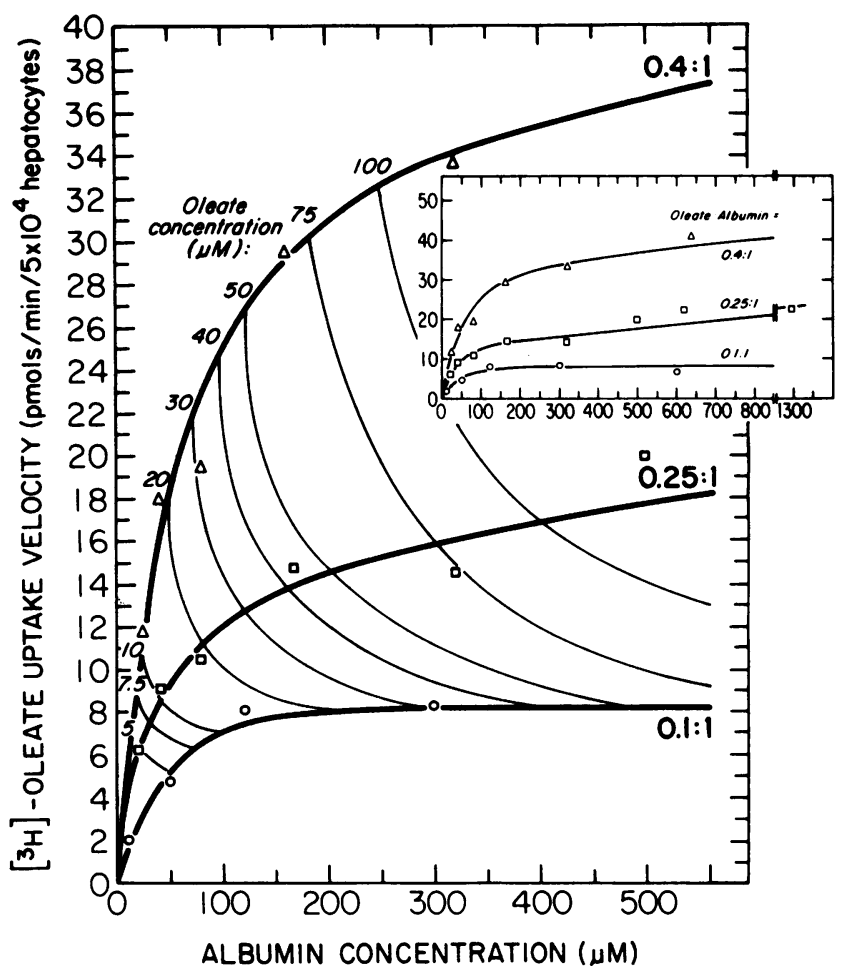

Figure 2. $\left[{ }^{3} \mathrm{H}\right]$ Oleate uptake velocity by isolated hepatocytes at different $\nu$ 's. The calculated equilibrium unbound oleate concentrations, in $\mu \mathrm{M}$, are: $0.031(\nu=0.4: 1), 0.0178(\nu=0.25: 1)$, and $0.0065(\nu$ $=0.1: 1)$. Were uptake driven solely by the unbound concentration, shifting from $0.4: 1$ to $0.25: 1$, from $0.25: 1$ to $0.1: 1$, and from $0.4: 1$ to $0.1: 1$ should decrease uptake by $43 \%, 64 \%$, and $79 \%$, respectively. The figure shows that this linear relationship between uptake and calculated unbound oleate does not apply at low oleate albumin concentrations. For example, at $10 \mu \mathrm{M}$ oleate (as indicated by the downwardly convex line connecting the three curves) uptake decreases by only $17 \%, 23 \%$, and $38 \%$ as $\nu$ decreases from $0.4: 1$ to $0.25: 1$, from $0.25: 1$ to $0.1: 1$, and from $0.4: 1$ to $0.1: 1$, respectively. The discrepancy between observed and expected uptake is progressively reduced at higher total oleate and albumin concentrations (see for example, 30 and $50 \mu \mathrm{M}$ oleate) until, at physiologic oleate and albumin levels, at which uptake values are maximal for a given value of $\nu$, observed and expected uptake rates resulting from changes in $\nu$ coincide: uptake decreases from $41.8 \pm 4.6$, to $21.4 \pm 1.7$, and $8.7 \pm 0.99 \mathrm{pmol} / \mathrm{min}$ per 50,000 cells at $\nu$ of $0.4: 1,0.25: 1$, and $0.1: 1$, respectively. The inset shows the same curves on a different scale.
Effects of differing substrate volumes. The basis for the discrepancy between uptake velocity and equilibrium unbound oleate concentration at low oleate/albumin concentrations remains to be explained. We postulated $(a)$ that the pseudosaturation curves illustrated in Fig. 1 reflected limited availability of substrate (i.e., unbound oleate) for uptake at the cell surface at low absolute oleate/albumin concentrations; and that $(b)$ increasing the volume of a given oleate/albumin substrate solution relative to a fixed volume of cell suspension might make more substrate available by one or more of several possible mechanisms, thereby increasing oleate uptake velocity at low absolute oleate/albumin concentrations without affecting oleate uptake at physiologic oleate/albumin concentrations. This was found to be the case. As illustrated in Fig. 5, for all three cell types studied, the " $K_{\mathrm{m}}$ " observed for a given $\nu$ was found to be inversely related to the volume of substrate solution added per unit volume of cell suspension, while the observed " $V_{\max }$ 's" were volume independent (Table II). Analogous results were obtained for at least two different $\nu$ for each cell type (data not shown).

\section{Discussion}

One of the oldest and most respected pair of assumptions in pharmacology and pharmacokinetics holds that, with respect to the cellular sequestration of protein bound ligands: $(a)$ the

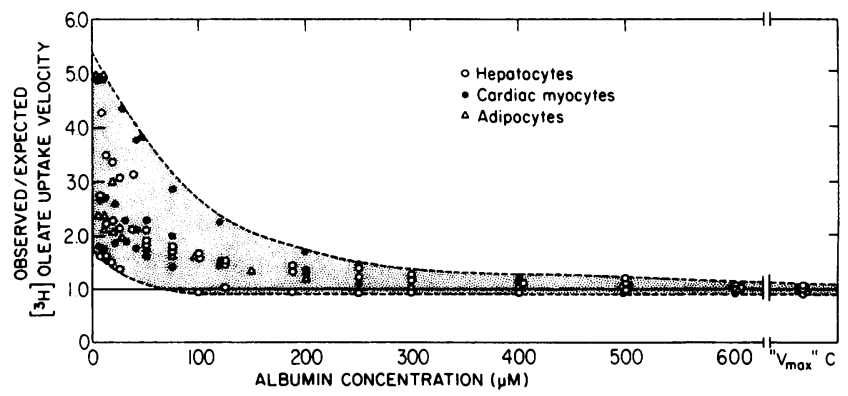

Figure 3. Effect of increasing albumin concentrations on the ratio of observed to expected $\left[{ }^{3} \mathrm{H}\right]$ oleate uptake for the three cell types. At low albumin concentrations the observed uptake is clearly greater than that expected on the basis of $\nu$ and the consequent unbound oleate concentration. However, the discrepancy is progressively reduced at albumin concentrations approaching physiologic levels until at albumin concentrations yielding the " $V_{\max }$ " values (" $V_{\max }$ " $\mathrm{C}$, see Fig. 1), the observed and expected uptake coincide (ratio $=1$ ). The data were calculated for two to three different $\nu$ per cell type as shown in Fig. 2. 

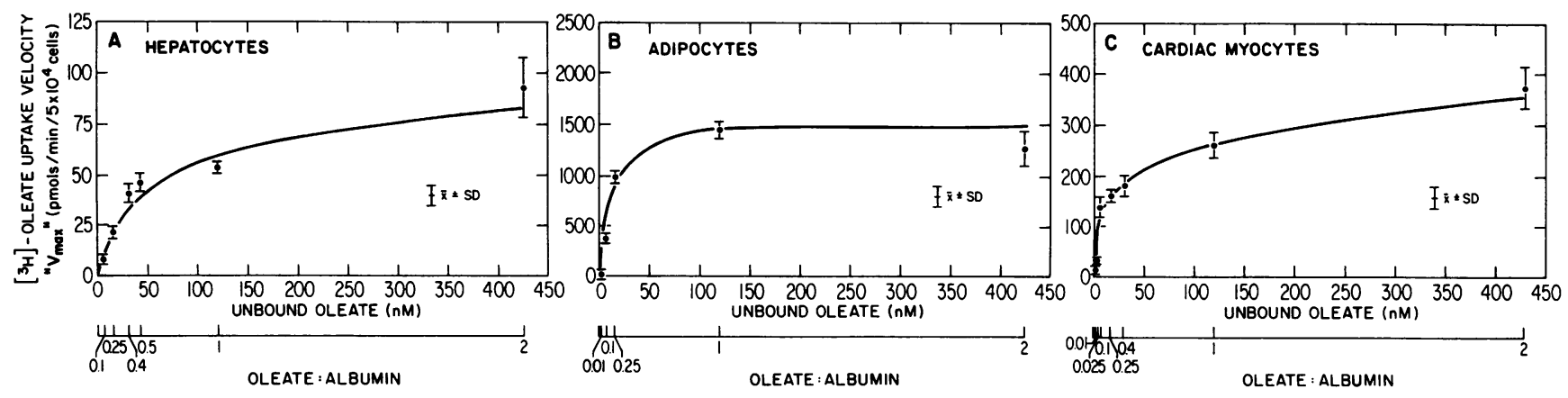

Figure 4. $\left[{ }^{3} \mathrm{H}\right]$ Oleate uptake (" $V_{\max }$ ” values, see Fig. 1 and Table I) by isolated hepatocytes $(A)$, adipocytes $(B)$, and cardiac myocytes $(C)$, as a function of $\nu$ and consequent oleate unbound concentration. For each cell type a rectangular hyperbola best describes the relationship between uptake values at physiologic concentrations of albumin and unbound oleate concentrations. Data are mean $\pm \operatorname{SD}(n=3-4)$.

unbound ligand fraction is the pool from which uptake occurs; and $(b)$ the bound fraction equilibrates virtually instantaneously with the unbound fraction, thus serving as a constant reservoir of substrate for future uptake. These two assumptions (7-9), are the essential basis for what is referred to as the "conventional model" for cellular uptake. Within the past 15 years, each of these assumptions has been challenged by experimental observations.

The equilibrium binding constants for the binding to albumin of such compounds as BSP and fatty acids were determined in the 1960s (10). At that time classic studies by Spector and associates (46) recognized that the numerous different classes of fatty acid binding sites on the albumin molecule, each with its own affinity for fatty acid molecules, served to buffer the concentration of unbound ligand in solutions containing both fatty acids and albumin. The equilibrium concentration of unbound ligand was determined principally by the molar ratio of fatty acid to albumin, and was, over a very wide range of concentrations, virtually independent of the absolute concentrations of either. Methodologic advances in the 1970s made it possible to measure the spontaneous unidirectional dissociation rates of the albumin complexes of compounds such as long chain fatty acids and bilirubin (51-53), thus obtaining more information about the kinetics of ligand/ protein interactions than had been obtainable from the previously available measurements of equilibrium association or dissociation constants. Hepatic physiologists subsequently pointed out (15) that these measured dissociation rates were too slow to provide sufficient unbound ligand to account for measured hepatic uptake rates of both fatty acids and bilirubin $(15,54,55)$.

In 1981 studies from two laboratories demonstrated that the uptake rates of fatty acids (13) and bile acids (14) by the isolated perfused rat liver did not correlate under a variety of experimental perturbations with the calculated concentration of unbound ligand. Analogous observations concerning bilirubin, BSP (16), rose bengal (56), and other ligands (19-25) were reported. When these data were considered in the light of the previously determined "off rates" for the corresponding ligand/albumin complexes (51-53), both groups concluded that bound as well as free ligand participated in the uptake process through interaction of ligand/albumin complexes with the cell surface, thereby amplifying and conceptualizing hypotheses previously suggested by Baker and Bradley (10), Bloomer et al. (11), and Anwer et al. (12). The precise mechanism for the surface-mediated dissociation of ligand/albumin complexes proposed by Forker and associates (14) was not defined, but a variety of alternative explanations for the kinetic observations, including nonequilibrium binding of ligand to albumin and the effects of an unstirred water layer in Disse's space were considered but rejected on the basis of detailed mathematical analysis (33). Weisiger, Gollan, and Ockner postulated the existence on the liver cell surface of a specific albumin receptor, which was presumed to facilitate ligand uptake by accelerating dissociation of ligand/albumin complexes, perhaps through a conformational change in the albumin molecule $(13,15)$. This
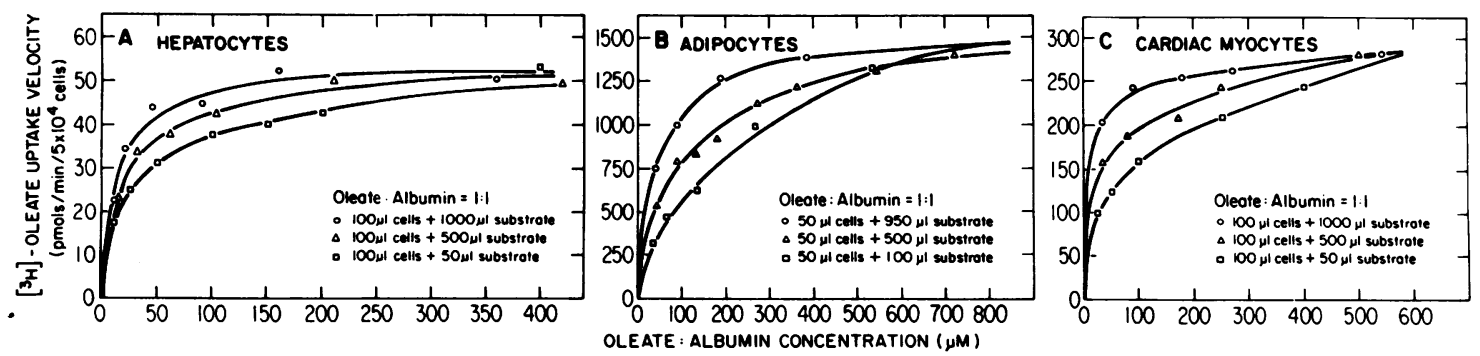

Figure 5. Substrate volume dependence of " $K_{\mathrm{m}}$ " for $\left[{ }^{3} \mathrm{H}\right]$ oleate uptake by isolated hepatocytes $(A)$, adipocytes $(B)$, and cardiac myocytes $(C)$ at $\nu=1: 1$ and different final total oleate/albumin concentrations. In the studies illustrated a fixed volume of cells ( $100 \mu l$ for hepatocytes and cardiac myocytes and $50 \mu \mathrm{l}$ for adipocytes) was added to increasing volumes of substrate $(50,500$, and 1,000 $\mu \mathrm{l}$ for hepatocytes and cardiac myocytes and 100,500 and $950 \mu \mathrm{l}$ for adipocytes) and the uptake determined as described in Methods. While " $V_{\max }$ " values were virtually identical, " $K_{\mathrm{m}}$ " values were inversely related to the volume of substrate added for each cell type (Table II). Qualitatively similar results were obtained for at least two different $\nu$ for each cell type. 


\begin{tabular}{|c|c|c|c|c|c|c|}
\hline \multirow[b]{2}{*}{ Substrate volume*: } & \multirow[b]{2}{*}{ Low } & \multirow[b]{2}{*}{ Medium } & \multirow[b]{2}{*}{ High } & \multicolumn{3}{|c|}{$P$ values } \\
\hline & & & & Low vs. med. & Med. vs. high & Low vs. high \\
\hline \multicolumn{7}{|l|}{ Hepatocytes } \\
\hline$" V_{\max } "$ & $50 \pm 3$ & $50 \pm 2$ & $53 \pm 3$ & NS & NS & NS \\
\hline$" K_{\mathrm{m}} "$ & $23 \pm 3$ & $16 \pm 1$ & $13 \pm 1$ & $<0.02$ & $<0.05$ & $<0.01$ \\
\hline \multicolumn{7}{|l|}{ Adipocytes } \\
\hline “ $V_{\max } "$ & $1,574 \pm 214$ & $1,471 \pm 58$ & $1,582 \pm 259$ & NS & NS & NS \\
\hline “ $K_{\mathrm{m}} "$ & $159 \pm 38$ & $83 \pm 9$ & $52 \pm 25$ & $<0.05$ & NS & $<0.02$ \\
\hline \multicolumn{7}{|c|}{ Cardiac myocytes } \\
\hline “ $V_{\max } "$ & $246 \pm 26$ & $261 \pm 16$ & $275 \pm 3$ & NS & NS & NS \\
\hline$" K_{\mathrm{m}} "$ & $44 \pm 12$ & $24 \pm 6$ & $12 \pm 1$ & NS & $<0.05$ & $<0.01$ \\
\hline
\end{tabular}

Uptake values shown in Fig. 5 were fitted to the Michaelis Menten equation as described in Methods. Data (mean \pm SD, $n=3$ ) are expressed as picomoles/minute per $5 \times 10^{4}$ cells (" $V_{\max }$ ") or $\mu \mathrm{M}$ oleate/albumin (" $K_{\mathrm{m}}$ "). * Respective low, medium and high substrate volumes for hepatocytes and cardiac myocytes were $50,500,1,000 \mu \mathrm{l}$ and for adipocytes $100,500,950 \mu \mathrm{l}$. Cell volume was constant in all experiments (100 $\mu \mathrm{l}$ for hepatocytes and cardiac myocytes and $50 \mu$ for adipocytes).

receptor was postulated in part because of studies which seemed to indicate that in perfusions employing various concentrations of $1: 1$ oleate/albumin complexes, oleate uptake was saturable at oleate concentrations that failed to produce saturation in the presence of a fixed albumin concentration (i.e., using variable $\nu$ ). In support of the albumin receptor and surface mediated dissociation hypotheses, albumin was reported to bind to liver cells and adipocytes with moderate affinity $(13,57)$. Other studies, however, failed to demonstrate specific binding of albumin to isolated rat hepatocyte plasma membranes or to identify in such membranes a protein with high affinity for albumin (26).

We $(23)$ and others $(19,58)$ have previously reproduced in isolated hepatocytes the kinetic observations initially reported in the perfused rat liver (13), thus demonstrating that they were not in some way dependent on an intact lobular architecture. Analogous results have been observed in hepatocyte monolayer cultures (21). They have also been reproduced with binding proteins such as $\beta$-lactoglobulin (23) and ligandin (28), demonstrating that they are not albumin specific. In the present report we find patterns of oleate uptake with respect to albumin concentration and $\nu$ in isolated rat adipocytes and cardiomyocytes virtually identical to those in hepatocytes, confirming observations in heart (25), brain (24), and uterus (22) that these kinetic patterns are not even liver specific, and that they occur in tissues in which, unlike the fenestrated hepatic sinusoidal endothelium, the continuous capillary endothelium poses a barrier to the extravascular diffusion of circulating ligand/albumin complexes. Most importantly, the data in Figs. 3 and 4 clearly indicate that at physiologic concentrations of albumin (3.5-5 g or 500-700 $\mu \mathrm{M})$, oleate uptake into cell suspensions of each of the cell types studied is a saturable function of the equilibrium unbound oleate concentration; i.e., at physiologic albumin concentrations the conventional model applies.

Whether these results, obtained in isolated cell suspensions, apply to intact tissues remains to be established. Our studies were conducted at nominal volumes of cells: fluid of up to $2: 1$ in contrast to ratios of 5-6:1 in intact liver, and possibly greater in myocardium and adipocytes. Although these differences may be offset by the fact that plasma in tissue is constantly replenished by perfusion, not static as in the present studies, replication of our studies in perfused organs would be of considerable interest.

Some comments regarding the choice of the data analysis employed in this study are necessary. The uptake data obtained at a fixed $\nu$ (Fig. 1) were fitted by a simple rectangular hyperbola for various reasons. First, such data have historically been analyzed by the Michaelis-Menten equation and interpreted as reflecting the saturation of an albumin receptor (see for example references 13 and 25). Second, the regression procedure provides a convenient method to describe the data from a phenomenological point of view; the experimental data in question are fully consistent with a rising portion followed by a final flat asymptote, although not distinguishable statistically from an alternative fit in which the asymptote has a small positive slope. For this reason, the final portion of the curves shown in Fig. 1 cannot, per se, be used to exclude the possibility that a small portion of oleate uptake is linearly dependent on albumin concentrations. At albumin concentrations of $\geq 500 \mu \mathrm{M}$ the upper bound of this component would be $\leq 10 \%$ of the total uptake for all three cell types at any $\nu$, as indicated by calculated deviations from a linear Augustinsson plot. In this albumin concentration range however, the percent deviation of the uptake data from the flat asymptote, while slightly positive, is not statistically different from zero $(10 \pm 16$, $8 \pm 12$, and $1 \pm 9 \%$ for hepatocytes, adipocytes, and cardiac myocytes, respectively). Thus, although we cannot exclude that at physiologic albumin concentrations oleate uptake may be in small measure dependent on albumin concentrations these data provide no definitive evidence for such a component, and suggest that, if present, it may be very small. Most importantly, we believe that the experimental data themselves are self-consistent, and can be interpreted independently of mathematical assumptions implicit in the data fitting procedure employed. This is best exemplified by an examination of Fig. 2; it is clear that the data per se indicate that at low oleate/albumin concentrations the uptake observed upon 
shifting from a given $\nu$ to a lower one is much greater than that expected on the basis of the new unbound oleate concentration. The discrepancy, however, diminishes with increasing oleate/albumin concentrations until it virtually disappears at physiologic or quasiphysiologic albumin concentrations. In fact, qualitatively and quantitatively similar results could be obtained by using the raw uptake data rather than the " $V_{\max }$ " at albumin concentrations $\simeq 600 \mu \mathrm{M}$. Thus, the ratio observed/expected oleate uptake is 1 (see Fig. 3) and likewise, a plot of the uptake data obtained at $600 \mu \mathrm{M}$ albumin vs. the respective unbound oleate concentrations also yields a rectangular hyperbola with resulting $V_{\max }$ and $K_{\mathrm{m}}$ values virtually identical to the ones obtained using the " $V_{\max }$ " values represented in Fig. 4 ( $V_{\max }: 113 \pm 13,1456 \pm 157$, and $424 \pm 22$ pico$\mathrm{mol} / \mathrm{min}$ per $5 \times 10^{4}$ cells, $K_{\mathrm{m}}: 84 \pm 14,21 \pm 4$, and $27 \pm 4 \mathrm{nM}$ for hepatocytes, adipocytes, and cardiac myocytes, respectively; compare with values given in Results).

That oleate uptake in adipocytes $(41,45)$, cardiac myocytes (44), and hepatocytes (59) is a saturable process with respect to the concentration of unbound fatty acid has, in fact, been documented in the years since 1981 (45); it could even be inferred from the data in the original publication proposing an albumin receptor (13) were the uptake values obtained at different $\nu$ plotted versus the unbound rather than the total oleate concentration (Fig. 6). Moreover, elegant competition studies by Goresky and colleagues (60-62) also indicate that fatty acid uptake in vivo is a function of the unbound rather than bound concentration. Fatty acid uptake by these various cell types appears on the basis of this and other published kinetic evidence $(44,45)$ to be carrier mediated and to involve a basic ( $\mathrm{pI}$ 9.1) 40-kD plasma membrane fatty acid binding protein $\left(\mathrm{FABP}_{\mathrm{PM}}\right)$, identified in liver (63), gut (64), adipose tissue (41), and heart $(44,65)$ and distinct both structurally and immunologically from the 12-14-kD cytosolic fatty acid binding proteins (FABP ${ }_{C}$ 's) described previously in these tissues (66). Nevertheless, the precise nature of the membrane carrier system(s) and its driving forces remain to be established $(67,68)$.

Virtually all the observations concerning fatty acid uptake not consistent with the conventional model have, in retrospect, been made at low, nonphysiologic concentrations of albumin $(13,21)$. In our own studies (Fig. 3), the lower the albumin concentration, the more the uptake velocities deviate from those predicted by the conventional model on the basis of the equilibrium unbound oleate concentrations. The physiologic significance of such observations is uncertain, however, they are reproducible and thus may require an explanation.

A partial explanation is suggested by the data in Fig. 5. The

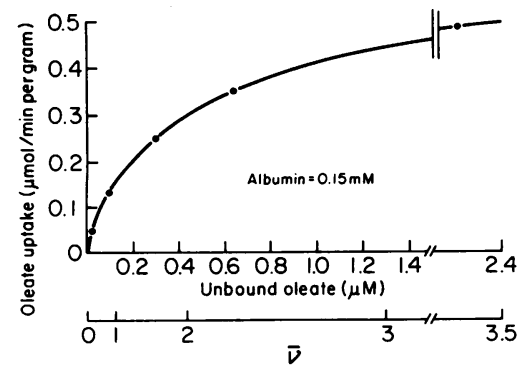

Figure 6. Oleate uptake by perfused rat liver as a function of different oleate:albumin ratios (v). This figure, redrawn from the original data by Weisiger et al. (reference 13) shows that oleate uptake is a saturable function of unbound oleate concentra-

tions provided that uptake is plotted against unbound rather than total oleate concentrations (for comparison, see Fig. 1 A, reference 13). (Reprinted from Science, 1981, 211:1048-1051, with permission. Copyright 1981 by the American Association for the Advancement of Science.). apparent saturation of oleate uptake that occurs (despite a constant equilibrium unbound oleate concentration) when the oleate concentration is increased at a fixed $\nu$ obviously does not reflect true saturation of an uptake mechanism. The " $K_{\mathrm{m}}$ ' $\mathrm{s}$ " of the pseudosaturation curves illustrated in Figs. 1 and 2 are a function of substrate availability: they decrease with increasing volumes of substrate added to a fixed number of cells (Fig. 5) and with a decreasing number of cells added to a fixed volume of substrate (data not shown). By contrast, the " $V_{\max }$ ' $s$ " of these same curves were independent of the substrate to cell volume ratio, and appear to reflect the capacity of the plasma membrane transport system to take up oleate at a given concentration of unbound ligand when substrate availability is not rate limiting.

If the substrate for fatty acid uptake is unbound ligand adjacent to the cell surface, two possible mechanisms have been proposed that might explain limited availability of substrate at low concentrations of albumin/ligand complexes in the bulk medium. The first is dissociation limited availability of unbound ligand, with resulting disequilibrium between bound and unbound ligand pools. Although more recent estimates of ligand/albumin dissociation rates (69) are appreciably faster than those reported a decade ago (53), detailed analyses by Jansen (35) and more recently by Weisiger (36), show that this possibility cannot be dismissed. Indeed, the mathematical model presented by Weisiger is generally consistent with our experimental data, in that it predicts that albumin concentrations govern the transition between "extrinsically limited" and "intrinsically limited" (i.e., between available substrate limited or membrane transport limited) uptake. However, whether the dependence of uptake on the cell to substrate volume ratio (Fig. 5) reflects dissociation limited uptake or some other phenomenon is uncertain, since substrate depletion in our studies never exceeded $5 \%$ even at the lowest volumes of substrate used. The second possible mechanism relates to the unstirred water layer which presumably surrounds every cell (70). Due to their small concentration, the flux of unbound ligands such as long chain fatty acids across a nonequilibrium atmosphere surrounding the cell surface may be smaller than that of albumin ligand complexes (34). A role for unstirred water layers in producing the observed kinetic effects, once dismissed (33), has been revived by a new and detailed mathematical analysis by Bass and Pond $(34,71,72)$. Indeed, these two possible mechanisms are not mutually exclusive. Both may contribute, along with some form of nonspecific surface-mediated dissociation process $(14,17,71,73)$ to the patterns of uptake kinetics observed at unphysiologically low concentrations of albumin. Indeed, our studies cannot rule out the possibility that such a mechanism may also exist at physiologic albumin concentrations. The nature of our data suggests that its contribution to oleate uptake under these conditions, if any, may be small.

Likewise, whether findings quantitatively similar to those reported in this paper apply to other classes of albumin bound ligands and to other settings cannot be inferred (74). However, various authors have recently offered explanations, also generally consistent with the conventional model, for apparent paradoxes in the hepatic uptake of different organic anions (75, 76). Furthermore, other studies suggest that changes in plasma albumin concentrations normally encountered in vivo may have little effect on the intrinsic elimination of drugs (77).

In summary, the present study clearly demonstrates that at physiologic concentrations of albumin and oleate, fatty acid uptake into isolated suspensions of hepatocytes, adipocytes, 
and cardiac myocytes is a saturable function of the concentration of unbound ligand, and that under these experimental conditions the conventional model is applicable. Alternative theories may explain deviations from the conventional model at very low albumin/ligand concentrations.

\section{Acknowledgments}

The authors wish to thank Ludvik Bass, Arthur A. Spector, and Decherd Stump for useful comments and Mary Barrett for preparation of the manuscript.

Supported in part by National Institutes of Health grant (AM-26438), grants from Assessorato Pubblica Istruzione, Regione Sardegna, Italy, and the New York Heart Association and gifts from the Polly Annenberg Levee Charitable Trust, and the Monique WeilCaulier Bequest.

\section{References}

1. Sorrentino, D., and P. D. Berk. 1988. Mechanistic aspects of hepatic bilirubin uptake. Semin. Liver Dis. 8:119-136.

2. Rose, C. P., and C. A. Goresky. 1977. Constraints on the uptake of labeled palmitate by the heart. Circ. Res. 41:534-565.

3. Rose, C. P., C. A. Goresky, and G. G. Bach. 1977. The capillary and sarcolemmal barriers in the heart: an exploration of labeled water permeability. Circ. Res. 41:515-533.

4. Goresky, C. A. 1977. Hepatic membrane carrier transport processes: their involvement in bilirubin uptake. In The Chemistry and Physiology of Bile Pigments. P. D. Berk and N. I. Berlin editors. U. S. Government Printing Office, Washington. 265-281.

5. Bernstein, L. H., J. Ben-Ezzer, L. Gartner, and I. M. Arias. 1966. Hepatic intracellular distribution of tritium-labeled unconjugated and conjugated bilirubin in normal and Gunn rats. J. Clin. Invest. 45:1194-1201.

6. Sorrentino, D., V. Licko, R. A. Weisiger, W. Bode, and B. Hoener. 1986. Early events in the hepatic transport of nitrofurazone. Clin. Res. 34:133. (Abstr.)

7. Brauer, R. W., and R. L. Pessotti. 1949. The removal of bromsulphthalein from blood plasma by the liver of the rat. J. Pharmacol. Exp. Ther. 97:358-370.

8. Grausz, H., and R. Schmid. 1971. Reciprocal relation between plasma albumin level and hepatic sulfobromophthalein removal. $N$. Engl. J. Med. 284:1403-1406.

9. Barnhart, J. L., and R. Clarenburg. 1973. Factors determining clearance of bilirubin in perfused rat liver. Am. J. Physiol. 225:497507.

10. Baker, J. K., and S. E. Bradley. 1966. Binding of sulfobromophthalein (BSP) sodium by plasma albumin. Its role in BSP extraction. J. Clin. Invest. 45:281-287.

11. Bloomer, J. R., P. D. Berk, J. Vergalla, and N. I. Berlin. 1973. Influence of albumin on the hepatic uptake of unconjugated bilirubin. Clin. Sci. 45:505-516.

12. Anwer, M. S., R. Kroker, and D. Hegner. 1976. Effect of albumin on bile acid uptake by isolated rat hepatocytes. Is there a common bile acid carrier? Biochem. Biophys. Res. Commun. 73:63-71.

13. Weisiger, R. A., J. Gollan, and R. Ockner. 1981. Receptor for albumin on the liver cell surface may mediate uptake of fatty acids and other albumin-bound substances. Science (Wash. DC). 211:10481051.

14. Forker, E. L., and B. A. Luxon. 1981. Albumin helps mediate removal of taurocholate by rat liver. J. Clin. Invest. 67:1517-1522.

15. Weisiger, R. A., J. L. Gollan, and R. Ockner. 1982. The role of albumin in hepatic uptake processes. In Progress in Liver Disease. H. Popper, F. Schaffner, editors. Grune \& Stratton, New York. 71-85.

16. Weisiger, R. A., J. Gollan, and R. Ockner. 1980. An albumin receptor on the liver cell may mediate hepatic uptake of sulfobromophthalein and bilirubin: Bound ligand, not free, is the major uptake determinant. Gastroenterology. 79:1065. (Abstr.)

17. Forker, E. L., and B. A. Luxon. 1983. Albumin-mediated trans- port of rose bengal by perfused rat liver. Kinetics of the reaction at the cell surface. J. Clin. Invest. 72:1764-1771.

18. Forker, E. L., B. A. Luxon, M. Snell, and W. O. Shurmantine. 1982. Effect of albumin binding on the hepatic transport of rose bengal: surface-mediated dissociation of limited capacity. J. Pharmacol. Exp. Ther. 223:342-347.

19. Barnhart, J. L., B. L. Witt, and W. G. Hardison. 1983. Uptake of iopanoic acid by isolated rat hepatocytes in primary culture. Am. J. Physiol. 244:G630-G636.

20. Blitzer, B. L., and L. Lyons. 1985. Enhancement of $\mathrm{Na}^{+}$-dependent bile acid uptake by albumin: direct demonstration in rat basolateral liver plasma membrane vesicles. Am. J. Physiol. 249:934938.

21. Fleischer, A. B., W. O. Shurmantine, B. A. Luxon, and E. L. Forker. 1986. Palmitate uptake by hepatocyte monolayers. Effect of albumin binding. J. Clin. Invest. 77:964-970.

22. Laufer, L. R., J. C. Gambone, G. Chaudhuri, W. M. Pardridge, and H. L. Judd. 1983. The effect of membrane permeability and binding by human serum proteins on sex steroid influx into the uterus. $J$. Clin. Endocrinol. Metabol. 56:1282-1287.

23. Nunes, R., C.-L. Kiang, D. Sorrentino, and P. D. Berk. 1988. "Albumin receptor" uptake kinetics do not require an intact lobular architecture and are not specific for albumin. J. Hepatol. 7:293-304.

24. Pardridge, W. M. 1984. Transport of nutrients and hormones through the blood-brain barrier. Fed. Proc. 43:201-204.

25. Rauch, B., C. Bode, H. M. Piper, J. F. Hutter, R. Zimmermann, E. Braunwell, W. Hasselbach, and W. Kubler. 1987. Palmitate uptake in calcium tolerant, adult rat myocardial single cells. Evidence for an albumin mediated transport across sarcolemma. J. Mol. Cell. Cardiol. 19:159-166.

26. Stremmel, W., B. J. Potter, and P. D. Berk. 1983. Studies of albumin binding to rat liver plasma membranes: implications for the albumin receptor hypothesis. Biochim. Biophys. Acta. 756:20-27.

27. Weisiger, R. A., C. M. Zacks, N. D. Smith, and J. L. Boyer. 1984. Effect of albumin binding on extraction of sulfobromophthalein by perfused elsamobranch liver: evidence for dissociation limited uptake. Hepatology. 4:492-501.

28. Stollman, Y. R., U. Gartner, L. Theilmann, N. Ohmi, and A. L. Wolkoff. 1983. Hepatic bilirubin uptake in the isolated perfused rat liver is not facilitated by albumin binding. J. Clin. Invest. 72:718-723.

29. Inoue, M., K. Okajima, S. Nagase, and Y. Morino. 1983. Plasma clearance of sulfobromophthalein and its interaction with hepatic binding proteins in normal and analbuminemic rats. Is plasma albumin essential for vectorial transport of organic anions in the liver? Proc. Natl. Acad. Sci. USA. 80:7654-7658.

30. Morgan, D. J., D. B. Jones, and R. A. Smallwood. 1985. Modeling of substrate elimination by the liver: has the albumin receptor model superseded the well-stirred model? Hepatology. 5:1231-1235.

31. Erlinger, S. 1986. Is serum albumin helpful for the hepatic uptake of taurocholate? Gastroenterology. 90:793-794.

32. Wolkoff, A. W. 1987. The role of an albumin receptor in hepatic organic anion uptake: the controversy continues. Hepatology. 7:777-779.

33. Forker, E. L., and B. A. Luxon. 1985. Effects of unstirred Disse fluid, nonequilibrium binding, and surface mediated dissociation on hepatic removal of albumin-bound organic anions. Am. J. Physiol. 248:709-717.

34. Bass, L., and S. M. Pond. 1988. The puzzle of cellular rates of uptake of protein bound ligands. In Pharmacokinetics: Mathematical and Statistical Approaches. A. Pecile and A. Rescigno, editors. Plenum Press, London. 241-265.

35. Jansen, J. A. 1981. Influence of plasma protein binding kinetics on hepatic clearance assessed from a "tube" model and a "well-stirred" model. J. Pharmacokinet. Biopharmacol. 9:15-26.

36. Weisiger, R. A. 1985. Dissociation from albumin: a potentially rate limiting step in the clearance of substances by the liver. Proc. Natl. Acad. Sci. USA. 82:1563-1567.

37. Van der Sluijs, P., B. Postema, and D. K. F. Meijer. 1987. 
Lactosylation of albumin reduces uptake rate of dibromosulfophthalein in perfused rat liver and dissociation rate from albumin in vitro. Hepatology. 7:688-695.

38. Sorrentino, D., C.-L. Kiang, and P. D. Berk. 1988. At physiologic albumin concentrations hepatocellular oleate uptake is driven by the equilibrium unbound oleate concentrations. Gastroenterology. 94:593. (Abstr.)

39. Sorrentino, D., C.-L. Kiang, R. Robinson, and P. D. Berk. 1988. "Albumin receptor" uptake kinetics occur in cardiac myocytes and adipocytes as well as hepatocytes and reflect dissociation limited availability of substrate. Hepatology. 8:1366 (Abstr.)

40. Stremmel, W., and P. D. Berk. 1986. Hepatocellular influx of $\left[{ }^{14} \mathrm{C}\right]$ oleate reflects membrane transport rather than intracellular metabolism or binding. Proc. Natl. Acad. Sci. USA. 83:3086-3090.

41. Schwieterman, W., D. Sorrentino, B. J. Potter, J. Rand, C.-L. Kiang, D. Stump, and P. D. Berk. 1988. Uptake of oleate by isolated rat adipocytes is mediated by a $40-\mathrm{kD}$ plasma membrane fatty acid binding protein closely related to that in liver and gut. Proc. Natl. Acad. Sci. USA. 85:359-363.

42. Moody, A. J., M. A. Stan, M. Stan, and J. Gleimann. 1974. A simple free fat cell bioassay for insulin. Horm. Metab. Res. 6:12-16.

43. Hewett, K., M. J. Legato, P. Danilo, and R. B. Robinson. 1983. Isolated myocytes from adult canine left ventricle: $\mathrm{Ca}^{2+}$ tolerance, electrophysiology and ultrastructure. Am. J. Physiol. 245:H830-H839.

44. Sorrentino, D., D. Stump, B. J. Potter, R. B. Robinson, R. White, C.-L. Kiang, and P. D. Berk. 1988. Oleate uptake by cardiac myocytes is carrier mediated and involves a $40-\mathrm{kD}$ plasma membrane fatty acid binding protein similar to that in liver, adipose tissue, and gut. J. Clin. Invest. 82:928-935.

45. Abumrad, N. A., R. H. Perkins, J. H. Park, and C. R. Park. 1981. Mechanism of long chain fatty acid permeation in the isolated adipocyte. J. Biol. Chem. 256:9183-9191.

46. Spector, A. A., K. John, and J. E. Fletcher. 1969. Binding of long chain fatty acids to bovine serum albumin. J. Lipid Res. 10:5667.

47. Spector, A. A., J. E. Fletcher, and J. D. Ashbrook. 1971. Analysis of long-chain free fatty acid binding to bovine serum albumin by determination of stepwise equilibrium constants. Biochemistry. 10:3229-3232.

48. Wosilait, W. D., and P. Nagy. 1976. A method of computing drug distribution in plasma using stepwise association constants. Clofibrate as an illustrative example. Comput. Programs Biomed. 6:142148.

49. Goodman, D. S. 1958. The distribution of fatty acids between n-heptane and aqueous phosphate buffer. J. Am. Chem. Soc. 80:3887-3892.

50. Berman, M. M., and M. F. Weiss. 1967. User's manual for SAAM (DHEW, GPO) Wash., DC. U. S. Public Health Service Publication 1703.

51. Svenson, A., E. Holmer, and L. Andersson. 1974. A new method for the measurement of dissociation rates for complexes between small ligands and proteins applied to the palmitate and bilirubin complexes with serum albumin. Biochim. Biophys. Acta. 342:54-59.

52. Faerch, T., and J. Jacobsen. 1975. Determination of association and dissociation rate constants for bilirubin-bovine serum albumin Arch. Biochem. Biophys. 168:351-357.

53. Scheider, W. 1978. Dissociation rate of serum albumin-fatty acid complex from stop-flow dielectric study of ligand exchange. Biophys. J. 24:260-262.

54. Goresky, C. A. 1964. Initial distribution and rate of uptake of sulfobromophthalein in the liver. Am. J. Physiol. 207:13-26.

55. Paumgartner, G., and J. Reichen. 1976. Kinetics of hepatic uptake of unconjugated bilirubin. Clin. Sci. Mol. Med. 51:169-176.

56. Forker, E. L., B. A. Luxon, and V. S. Sharma. 1985. Hepatic transport and binding of rose bengal in the presence of albumin and gamma globulin. Am. J. Physiol. 248:G702-G708.

57. Brandes, R. B., R. K. Ockner, R. A. Weisiger, and N. Lysenko. 1982. Specific and saturable binding of albumin to rat adipocytes: modulation by epinephrine and possible role in free fatty acid transfer. Biochem. Biophys. Res. Commun. 105:821-827.

58. Smith, D. J., M. Grossbard, E. R. Gordon, and J. L. Boyer. 1987. Taurocholate uptake by isolated skate hepatocytes: effect of albumin. Am. J. Physiol. 252:G479-G484.

59. Stremmel, W., G. Strohmeyer, and P. D. Berk. 1986. Hepatocellular uptake of oleate is energy dependent, sodium-linked, and inhibited by an antibody to a hepatocyte plasma membrane fatty acid binding protein. Proc. Natl. Acad. Sci. USA. 83:3584-3588.

60. Goresky, C. A., G. G. Bach, D. S. Daly, S. Mishkin, and I. M. Arias. 1978. Uptake of labeled palmitate by the intact liver: role of intracellular binding sites. Am. J. Physiol. 234:E542-E553.

61. Goresky, C. A., A. W. Wolkoff, C. P. Rose, and D. Cousineau. 1985. Sequestered tracer outflow recovery in multiple indicator dilution experiments. Hepatology. 5:805-814.

62. Goresky, C. A., G. G. Bach, C. P. Rose, and A. W. Wolkoff. 1986. Correspondence: The exponential extrapolation area test: no surprises. Hepatology. 6:545-546.

63. Stremmel, W., G. Strohmeyer, F. Borchard, S. Kochwa, and P. D. Berk. 1985. Isolation and partial characterization of a fatty acid binding protein from rat liver plasma membranes. Proc. Natl. Acad. Sci. USA. 82:4-8.

64. Stremmel, W., G. Lotz, G. Strohmeyer, and P. D. Berk. 1985. Identification, isolation, and partial characterization of a fatty acid binding protein from rat jejunal microvillous membranes. J. Clin. Invest. 75:1068-1076.

65. Potter, B. J., D. Stump, W. Schwieterman, D. Sorrentino, N. Jacobs, C.-L. Kiang, J. H. Rand, and P. D. Berk. 1987. Isolation and partial characterization of plasma membrane fatty acid binding proteins from myocardium and adipose tissue and their relationship to analogous proteins in liver and gut. Biochem. Biophys. Res. Commun. 148:1370-1376.

66. Ockner, R. K. 1986. Cellular fatty acid binding proteins: evolving concepts of structure and function. Hepatol. Rapid. Lit. Rev. 16:VII-XV.

67. Sorrentino, D., W. Schwieterman, C.-L. Kiang, T. McHugh, and P. D. Berk. 1987. The hepatocellular uptake of free fatty acids does not involve a sodium-fatty acid symport. Hepatology. 7:1036. (Abstr.)

68. Weisiger, R. A., J. G. Fitz, and B. F. Scharschmidt. 1987. Hepatic oleate uptake: electrochemical driving forces. Clin. Res. 35:416a. (Abstr.)

69. Weisiger, R. A., and W.-L. Ma. 1987. Uptake of oleate from albumin solutions by rat liver. Failure to detect catalysis of the dissociation of oleate from albumin by an albumin receptor. J. Clin. Invest. 79:1070-1077.

70. Barry, P., and J. Diamond. 1984. Effects of unstirred layers on membrane phenomena. Physiol. Rev. 64:763-782.

71. Forker, E. L., and C. Ghiron. 1988. ESR, albumin, and the riddle of organic anion uptake by the liver. Am. J. Physiol. 254:G463G464.

72. Weisiger, R. A., S. M. Pond, and L. Bass. 1988. Albumin modulates the unidirectional fluxes of oleate across a lipid water interface without altering the flux ratio. Hepatology. 8:1264. (Abstr.)

73. Horie, T., T. Mizuma, S. Kasai, and S. Awazu. 1988. Conformational change in plasma albumin due to interaction with isolated rat hepatocyte. Am. J. Physiol. 254:G465-G470.

74. Roda, E., R. Aldini, and A. Roda. 1987. Role of albumin in liver uptake. Gastroenterology. 92:1097-1098.

75. Smallwood, R. H., D. J. Morgan, G. W. Mihaly, and R. A. Smallwood. 1988. Lack of linear correlation between hepatic ligand uptake rate and unbound ligand concentration does not necessarily imply receptor-mediated uptake. J. Pharmacokinet. Biopharm. 16:397-411.

76. Tokumo, H., D. Mancuso, N. Busch, and R. T. Holzbach. 1988. Hydrophobicity is an important mechanism in hepatic uptake of organic anions. Hepatology. 8:1265. (Abstr.)

77. Oie, S., and Y. H. Yang. 1988. Effect of altered albumin concentrations on elimination of unbound prazosin in vivo in the rat and in the isolated perfused rat liver. J. Pharmacol. Sci. 77:948-951. 